\title{
FORCE PREVALENCE ANALYSIS
}

Author: Mr. Alejandro Schaller

\begin{abstract}
The electric field is enormously more powerful than the gravitational field. However, in our normal experience there is no electric field, the electric force is missing. How is it that when you get to larger and larger scales the gravitational field is extremely important whereas the electric field becomes less and less important [1]? This paper proposes a model to explain why gravitation dominates at a larger scale.
\end{abstract}

Key Words: Field, gravitation, electricity, unification, force, theory.

Author contact details: Email alejandroschaller@ frbb.utn.edu.ar WhatsApp: +5492914739920

Author Affiliation: National Technologic University - https://frbb.utn.edu.ar - 11 de Abril 461 - B8000LMI Bahía Blanca - Buenos Aires - Argentina - Telephone Number +54 2914555220

\section{Introduction}

This paper considers Dr Harishanker Ramachandran's lecture on electromagnetic field for EEE students [1] in which he shows a model proposed to explain why gravitation dominates at a larger scale.

The model shown on the lecture consists of a system formed by:

- a set of two charges (of different sign) close together, and

- $\quad$ another charge far away.

The charge that is far away would exert an attraction force over one of the charges (of the set of two charges) and a repulsion force over the other charge. Consequently, there would be very slight resultant force acting on the set of charges. After performing the calculation, it is deduced on the lecture that the resultant force is still too large to be consistent with a gravitational force.

I considered this model and thought of making two extra assumptions:

- that the set of the two charges are not fixed but rotating (as electron spins around a proton), and

- that the charge far away has a magnitude equal to the charge of the Earth.

After performing the calculation taking into account these assumptions, the resultant force showed to be consistent with a gravitational force.

\subsection{Different orders of magnitude of electrical and gravitational force}

We can compare the electric force with the gravitational force. Suppose that we have two protons separated by a distance " $\mathrm{d}$ ". The force due to gravity is the gravitational constant times the mass of the proton squared, divided by $d^{2}\left(F_{G}=\frac{G \cdot m_{p}^{2}}{d^{2}}\right)$. This is the force by means of which one proton attracts another proton, the "gravitational attraction". Whereas electrical interaction is: $F_{E}=\frac{1}{4 . \pi \cdot \varepsilon_{0}} \frac{e^{2}}{d^{2}}$

We can work out how much stronger the electric field is compared to the gravitational field dividing $F_{E}$ by $F_{G}$ (the $d^{2}$ will cancel out): 


$$
\frac{F_{E}}{F_{G}}=\frac{\frac{e^{2}}{4 \cdot \pi \cdot \varepsilon_{0}}}{G \cdot m_{p}^{2}}=\frac{\frac{\left(1.6 \times 10^{-19}\right)^{2}}{4 \cdot \pi \cdot 8.85 \times 10^{-12}}}{6.7 \times 10^{-11} \cdot\left(1.67 \times 10^{-27}\right)^{2}}=1.2 \times 10^{36}
$$

This result shows that the electric force between two protons is 36 orders of magnitude stronger than the gravitational force between those protons [1]. The force due to electricity is about a billion-billionbillion-billion time stronger than the force due to gravitation [2].

\section{Analysis of different models}

\subsection{Set of two charges close together and another charge far away, as shown on the lecture [1]:}

If there is an electron and a proton close to each other, and there is a positive charge far away, the electron is attracting the charge and the proton is repelling the charge, and this would result in very slight force acting on it. All these huge forces that are being exerted by the set of two charges would cancel. Let's work out what happens in such a case.

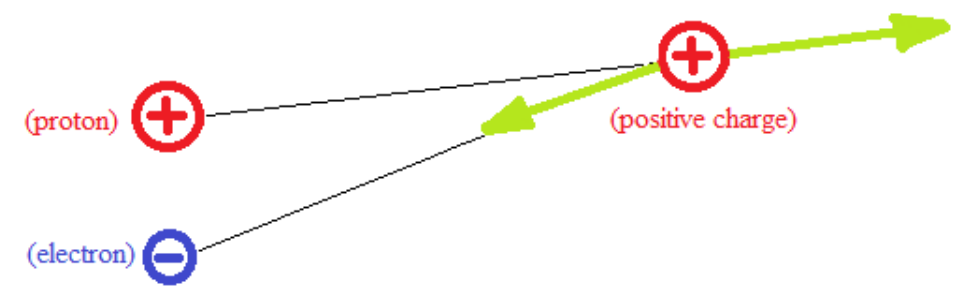

Figure 1. Set of two charges close together, and another charge far away.

Suppose that there is a charge $+Q$ and a charge $-Q$ separated by a distance $d$, as shown in figure 2. We want to know the field due to this charge combination, far away. We draw a straight line from $+\mathrm{Q}$ to the point " $\mathrm{p}$ " and the force will be away from $+\mathrm{Q}$. Then we draw another straight line connecting -Q to the point "p" and the force will be towards -Q; the forces mostly cancel.

However, if we write down what the forces are we can see that the distance from the point " $\mathrm{p}$ " to $-\mathrm{Q}$ is a larger distance than from the point " $\mathrm{p}$ " to $+\mathrm{Q}$. How much larger? We can draw perpendiculars and see that the distance $-\mathrm{Q}$ to " $\mathrm{p}$ " is equal to the distance $+\mathrm{Q}$ to "p" plus a little extra. To find out how much is that little extra we can see from the picture that it is " $d$. $\sin \theta$ ". So, the distance from $-\mathrm{Q}$ to " $\mathrm{p}$ " would be the distance from +Q to "p" plus $d \cdot \sin \theta$.

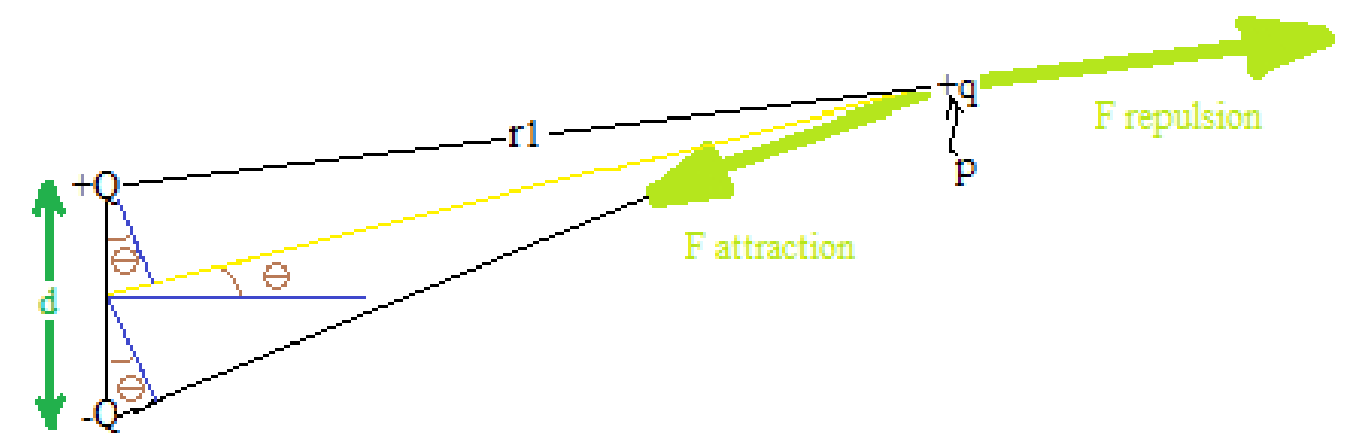

Figure 2. Analysis of the model as on the lecture 
If we want to find out what the force along the radius is, we have to subtract both forces. Supposing there is a charge $+q$ at the point " $p$ " and that the distance from $+Q$ to " $p$ " is $r_{1}$ :

$$
F_{r}=\frac{1}{4 \cdot \pi \cdot \varepsilon_{0}}\left(\frac{Q}{r_{1}^{2}}-\frac{Q}{\left(r_{1}+d \cdot \sin \theta\right)^{2}}\right) \cdot q
$$

Because we are looking from far away, $r_{1}$ is much larger than " $\mathrm{d}$ ". So we can approximate $\left(r_{1}+d \cdot \sin \theta\right)^{2}$ to $r_{1}^{2}+2 \cdot d \cdot \sin \theta \cdot r_{1}$ (neglecting the $\mathrm{d}^{2} \sin ^{2} \theta$ term).

$$
\begin{gathered}
r_{1} \gg d \therefore\left(r_{1}+d \cdot \sin \theta\right)^{2} \cong r_{1}^{2}+2 \cdot d \cdot \sin \theta \cdot r_{1} \\
F_{r}=\frac{Q \cdot q}{4 \cdot \pi \cdot \varepsilon_{0}} \frac{1}{r_{1}^{2}}\left(1-\frac{1}{1+\frac{2 \cdot d \cdot \sin \theta}{r_{1}}}\right)
\end{gathered}
$$

$\frac{2 . d . \sin \theta}{r_{1}}$ is a very small quantity because $d$ is small compared to $r_{1}$, so we can expand $1+\frac{2 . d \cdot \sin \theta}{r_{1}}$ and take it to the numerator: $F_{r}=\frac{Q \cdot q}{4 . \pi \cdot \varepsilon_{0}} \frac{1}{r_{1}^{2}}\left(1-\left(1-\frac{2 \cdot d \cdot \sin \theta}{r_{1}}\right)\right.$

The "1" will cancel out. We are left with: $F_{r}=\frac{Q \cdot q}{4 \cdot \pi \cdot \varepsilon_{0}} \frac{1}{r_{1}^{3}} \cdot 2 \cdot d \cdot \sin \theta$.

We know that $F_{r}=\frac{Q . q}{4 . \pi \cdot \varepsilon_{0}} \frac{1}{r_{1}^{2}}$ is Coulomb's law. So we have and extra term of: $\frac{2 . d . \sin \theta}{r_{1}}$

This means that Coulomb's law is reduced by this factor. Finally, we arrive at:

$$
F_{r}=\left(\frac{2 \cdot d \cdot \sin \theta}{r_{1}}\right) \quad . F_{\text {Coulomb }}
$$

Does this explain why gravitation dominates? $\mathrm{d}$ is of the order of $10^{-10} \mathrm{~m} . r_{1}$ is considered on the lecture as $1 \mathrm{~m}$. This gives a factor of $10^{-10}$ which could reduce $10^{36}$ to $10^{26}$, but it still is a very large number to be consistent with a gravitational force.

This means that having a set of two charges close together and another charge far away does not explain why gravitation dominates at a larger scale. 


\subsection{Same case as before, with two extra assumptions:}

\section{Assumptions:}

- that the set of charges are not fixed, but rotating (as electron spins around a proton), and

- that the far away charge has a magnitude equal to the charge of the Earth.

If the set of two charges is not fixed but the electron is spinning around the proton, there would be a net attractive force whenever the electron is closer than the proton to the far away positive charge (as shown in Figure 3a), and there would be a net repulsive force whenever the proton is closer than the electron to the far away positive charge (as shown in Figure 3b). Let's work out what happens in such a case, considering the net force as the radial force exerted by the far away positive charge over the set of two charges (a proton and its spinning electron). The proton has no velocity, so there is no magnetic force acting on it. We assume that the magnetic force over the electron cancels by pairs every time it occupies a symmetric position around the orbital, so that on average there would be no magnetic force.

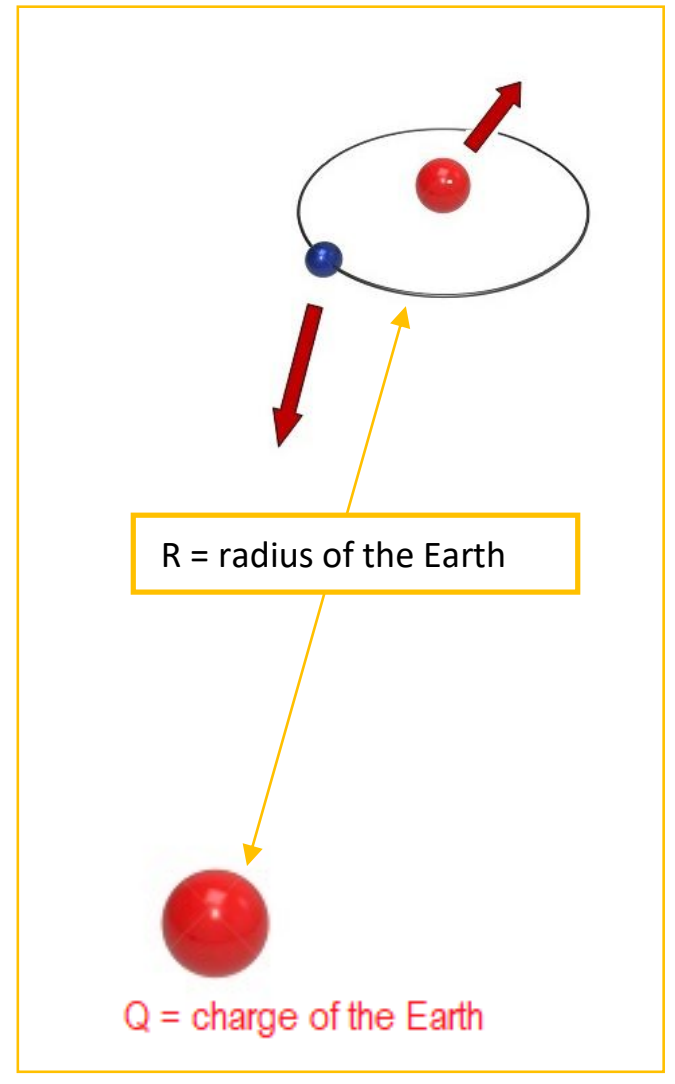

Figure 3a

Electron closer than proton

(to the far away charge " $Q$ ")

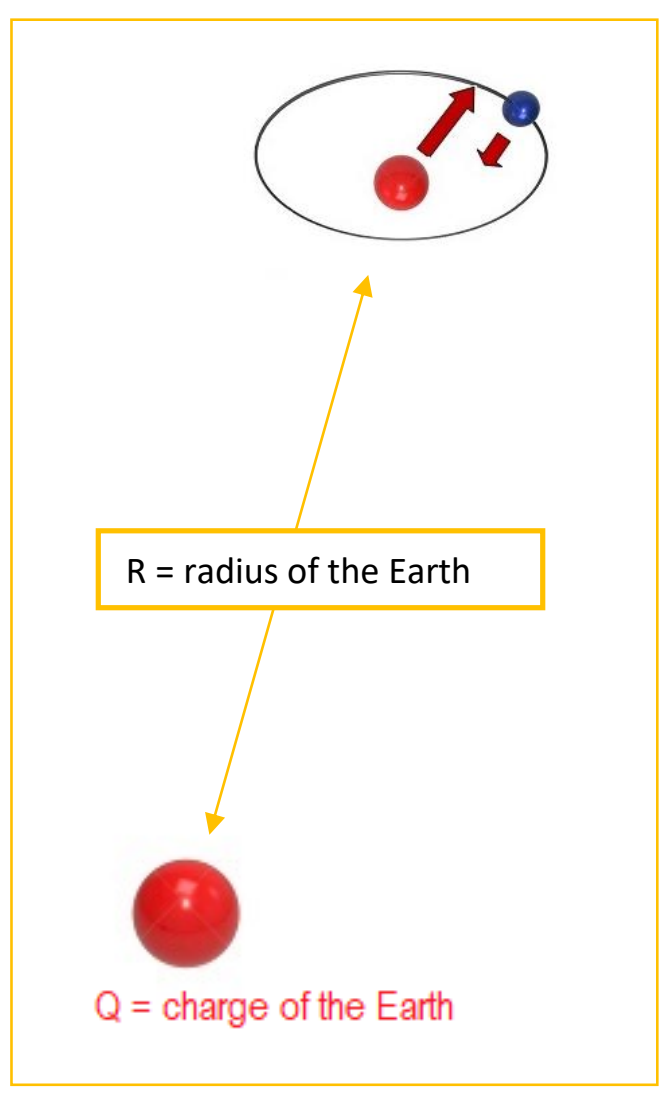

Figure 3b

Proton closer than electron (to the far away charge " $Q$ ") 
Suppose that there is a charge $-\mathrm{q}$ (electron) orbiting a charge $+\mathrm{q}$ (proton) at a distance $\mathrm{r}$, as shown in figure 4, and there is a positive charge (+Q) located far away from $+\mathrm{q}$, at a distance R equal to the Earth's radius. The radial distance from $+\mathrm{Q}$ to $-\mathrm{q}$ is approximately equal to $R+r \cdot \cos \varphi$.

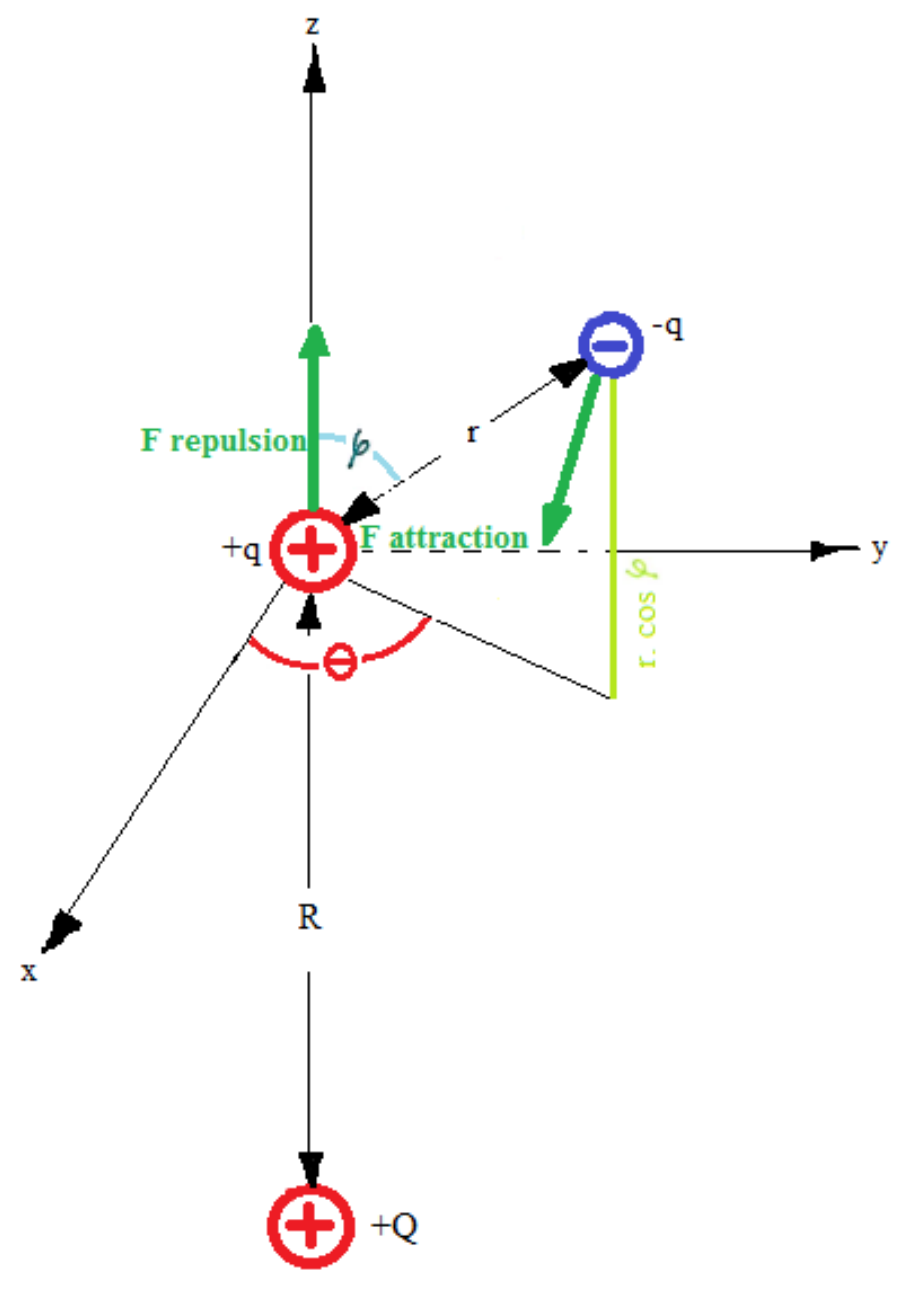

Figure 4. Analysis of the model with the two assumptions

$+\mathrm{Q}$ is exerting and attractive force over $-\mathrm{q}$ and a repulsive force over $+\mathrm{q}$. If we subtract both forces, we'll get an approximation of the resultant radial force:

$$
F_{r}=\frac{1}{4 \cdot \pi \cdot \varepsilon_{0}}\left(\frac{Q}{(R+r \cdot \cos \varphi)^{2}}-\frac{Q}{R^{2}}\right) \cdot q
$$

This is an instant force, as the electron orbits the proton the force changes due to the change of position of the electron. To calculate the average force, we'll have to integrate $F_{r}$ for every point on the surface of the orbital and then divide the result by the magnitude of the surface of integration itself. 
The electron is orbiting the proton at a distance " $\mathrm{r}$ " which is given by quantum dynamics; the radius of the orbitals can only adopt definite magnitudes and for this calculation Bohr's first orbital radius is used: $r=0.0529 \mathrm{~nm}$ [3].

In order to calculate the integration of $F_{r}$ over the orbital's surface, spherical coordinates are used. Integration limits will correspond to a sphere or radius " $r$ " centered on the proton:

$$
\theta=[0,2 \pi] \quad \varphi=[0, \pi]
$$

The surface differential element "ds" is shown shaded in green on Figure 5.

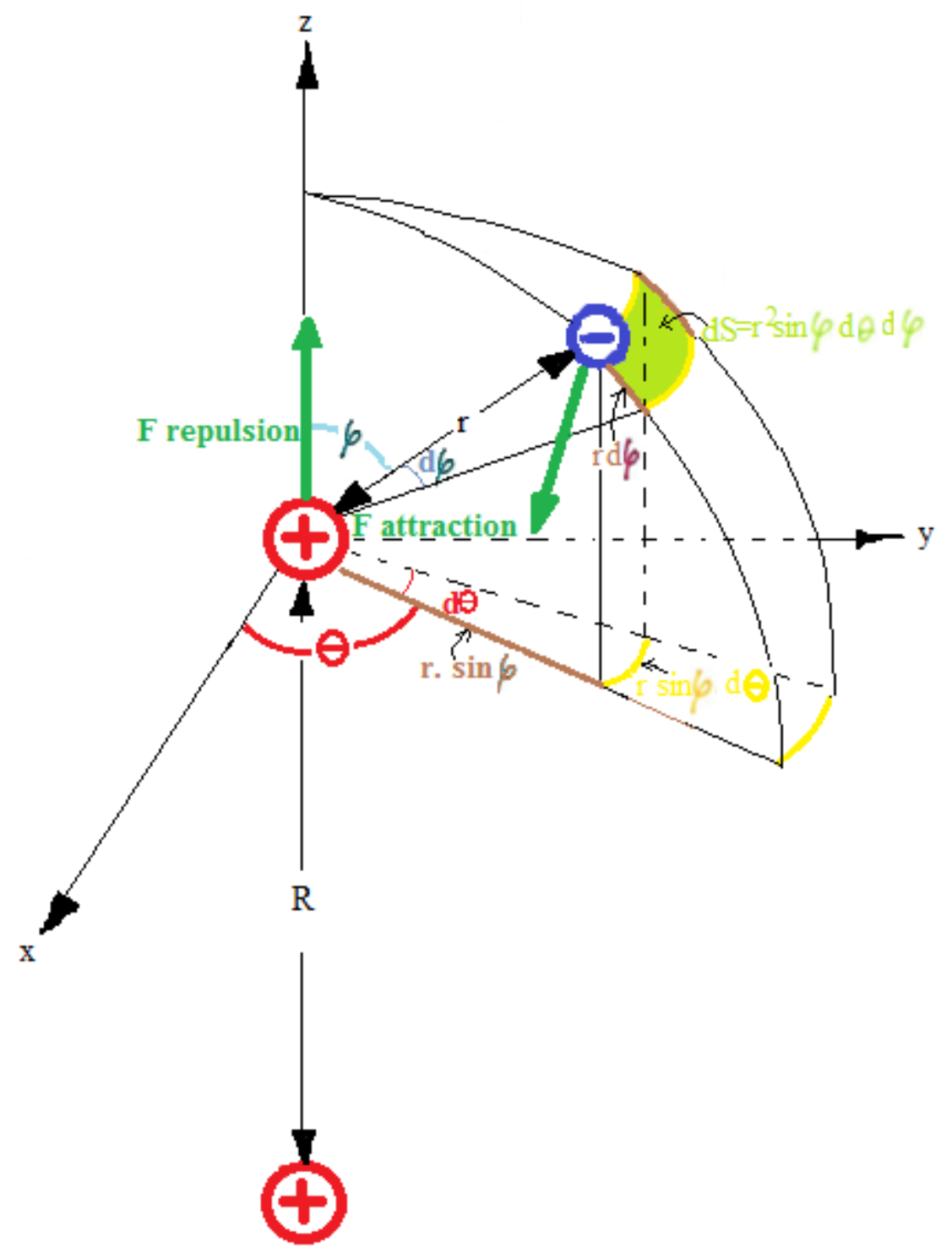

Figure 5. Graphic showing the surface differential element "dS" shaded in green. 


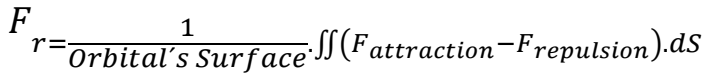

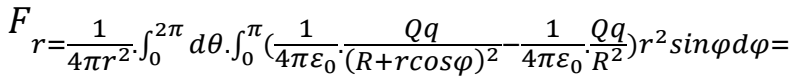

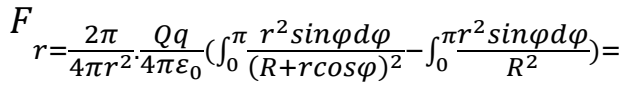

For the first integral the following substitution is used:

$$
\begin{aligned}
& \mathrm{u}=R+r \cos \varphi \quad \therefore \mathrm{du}=-r \sin \varphi d \varphi \quad \int \frac{-r d u}{u^{2}}=\frac{r}{u}=\frac{r}{R+r \cos \varphi} \\
& F_{r}=\frac{1}{2 r^{2}} \frac{Q q}{4 \pi \varepsilon_{0}}\left[( \frac { r } { R + r \operatorname { c o s } \varphi } ) \left\{\begin{array}{l}
\pi \\
0
\end{array}-\frac{r^{2}}{R^{2}} \cdot(-\cos \varphi)\left\{\begin{array}{l}
\pi \\
0
\end{array}\right]\right.\right. \\
& F_{r}=\frac{Q q}{4 \pi \varepsilon_{0}} \frac{1}{2 r^{2}}\left[\frac{r}{R-r}-\frac{r}{R+r}+\frac{r^{2}}{R^{2}} \cdot(-1-1)\right] \\
& F_{r}=\frac{Q q}{4 \pi \varepsilon_{0}} \frac{1}{2 r^{2}}\left[\frac{r(R+r-(R-r))}{(R-r)(R+r)}-\frac{2 r^{2}}{R^{2}}\right] \\
& F_{r}=\frac{Q q}{4 \pi \varepsilon_{0}} \frac{1}{2 r^{2}}\left[\frac{r 2 r}{R^{2}-r^{2}}-\frac{2 r^{2}}{R^{2}}\right] \\
& F_{r}=\frac{Q q}{4 \pi \varepsilon_{0}}\left[\frac{1}{R^{2}-r^{2}}-\frac{1}{R^{2}}\right] \\
& F_{r}=\frac{Q q}{4 \pi \varepsilon_{0}} \frac{1}{R^{2}}\left[\frac{1}{\left(1-\frac{r^{2}}{R^{2}}\right)}-1\right] \\
& F_{r}=\frac{Q q}{4 \pi \varepsilon_{0}} \frac{1}{R^{2}}\left[\frac{1}{\frac{R^{2}-r^{2}}{R^{2}}}-1\right] \\
& F_{r}=\frac{Q q}{4 \pi \varepsilon_{0}} \frac{1}{R^{2}}\left[\frac{R^{2}}{R^{2}-r^{2}}-1\right] \\
& F_{r}=\frac{Q q}{4 \pi \varepsilon_{0}} \frac{1}{R^{2}}\left[\frac{R^{2}-\left(R^{2}-r^{2}\right)}{R^{2}-r^{2}}\right] \\
& F_{r}=\frac{Q q}{4 \pi \varepsilon_{0}} \frac{1}{R^{2}}\left[\frac{r^{2}}{R^{2}-r^{2}}\right]
\end{aligned}
$$

We know that $F_{r}=\frac{Q . q}{4 . \pi \cdot \varepsilon_{0}} \frac{1}{R^{2}}$ is Coulomb's law. So, there is an extra term of: $\frac{r^{2}}{R^{2}-r^{2}}$ 
This means that Coulomb's law is reduced by this factor:

$$
F_{r}=\left(\frac{r^{2}}{R^{2}-r^{2}}\right) \cdot F_{\text {Coulomb }} \cong\left(\frac{r^{2}}{R^{2}}\right) \cdot F_{\text {Coulomb }}
$$

Does this explain why gravity dominates? $\quad \mathrm{r}$ is $0.529 \times 10^{-10} \mathrm{~m}, \mathrm{R}$ is $6370000 \mathrm{~m}$, this gives a factor $\frac{r^{2}}{R^{2}}=\frac{\left(0.529 \times 10^{-10}\right)^{2}}{6370000^{2}}=6.89 \times 10^{-35}$ which could reduce $1.2 \times 10^{36}$ almost completely.

This means that having a set of two charges (electron spinning around a proton) located at a distance equal to the radius of the Earth from a charge equal in magnitude to the charge of the Earth (situated over the surface of the Earth) could explain why gravitation dominates at a larger scale.

\section{Conclusion}

This paper considers a model proposed by Dr Harishanker Ramachandran to explain why gravitation dominates at a larger scale (a set of two charges of different sign close together, and another charge far away) and added two assumptions to that model (that the set of charges are not fixed but rotating, as electron spins around a proton, and that the far away charge has a magnitude equal to the charge of the Earth). The calculated result is consistent with a gravitational force.

\section{Appreciation}

The author would like to express his gratitude to José Luis Rodriguez (professor and doctor of Chemistry at the UNS and professor of Investigation Methodology at ESOA), for his supportive guidance.

\section{References}

1. Dr. Harishanker Ramachandran. Electromagnetic Field for EEE Students. Lecture 3. Coulomb's Law [online]. http://www.nptel.ac.in/courses/108106073/3. Checked: 04 May 2018.

2. Feynman Richard P. (2011). The Feynman Lectures on Physics Vol II: Mainly Electromagnetism and Matter. The New Millennium Edition. 1-1. Basic Books, New York. [online]: http://www.feynmanlectures.caltech.edu/II_01.html Checked: 23 May 2019.

3. Tipler Paul A. (1996). Física. Third Edition. 1159. Editorial Reverté, Spain. 